\title{
MULTIPLE SOLUTIONS FOR RESONANT ELLIPTIC SYSTEMS VIA REDUCTION METHOD
}

\author{
MARCELO F. FURTADO ${ }^{\bowtie}$ and FRANCISCO O. V. DE PAIVA
}

\author{
(Received 1 November 2009)
}

\begin{abstract}
We establish the existence of two nontrivial solution for some elliptic systems. In the proofs we apply variational methods and Morse theory.
\end{abstract}

2000 Mathematics subject classification: primary 35J20; secondary 35J50, 58E05.

Keywords and phrases: resonant elliptic systems, variational methods, Morse theory.

\section{Introduction}

We consider the gradient system

$$
\begin{cases}-\Delta u=F_{u}(x, u, v) & \text { in } \Omega, \\ -\Delta v=F_{v}(x, u, v) & \text { in } \Omega, \\ u=v=0 & \text { on } \partial \Omega,\end{cases}
$$

where $\Omega \subset \mathbb{R}^{N}$ is a bounded smooth domain, $N \geq 3$ and $F \in C^{2}\left(\Omega \times \mathbb{R}^{2}, \mathbb{R}\right)$ satisfies the subcritical growth condition

$$
\begin{aligned}
& \text { there exist } c_{1}>0 \text { and } 2<p<2 N /(N-2) \text { such that } \\
& |\nabla F(x, z)| \leq c_{1}\left(1+|z|^{p-1}\right) \quad \text { for }(x, z) \in \mathbb{R}^{N} \times \mathbb{R}^{2} .
\end{aligned}
$$

In order to state the other assumptions on $F$, let $\mathcal{M}_{2}(\Omega)$ denote the set of all continuous, cooperative and symmetric matrices of order two. More specifically, $A \in \mathcal{M}_{2}(\Omega)$ if it has the form

$$
A(x)=\left(\begin{array}{ll}
a(x) & b(x) \\
b(x) & c(x)
\end{array}\right)
$$

The first author was partially supported by CNPq/Brazil.

(C) 2010 Australian Mathematical Publishing Association Inc. 0004-9727/2010 \$16.00 
with $a, b, c \in C(\bar{\Omega}, \mathbb{R})$ and $b(x) \geq 0$ for all $x \in \bar{\Omega}$. Given $A \in \mathcal{M}_{2}(\Omega)$, we shall consider the interaction between the nonlinearity $F$ and the eigenvalues of the weighted linear problem

$$
\begin{cases}-\Delta\left(\begin{array}{l}
u \\
v
\end{array}\right)=\lambda A(x)\left(\begin{array}{l}
u \\
v
\end{array}\right) & \text { in } \Omega \\
u=v=0 & \text { in } \partial \Omega .\end{cases}
$$

In what follows, $\lambda_{k}(A)$ denotes the $k$ th positive eigenvalue of $(L P)$ (see Section 2 for more details). For simplicity of the statements we set $\lambda_{0}(A)=-\infty$.

We first notice that, if $\nabla F(x, 0,0) \equiv 0$, the problem $(P)$ possesses the trivial solution $(u, v)=(0,0)$. In this case the key point is to assure the existence of nontrivial solutions and therefore we need to introduce a condition that gives us information about the behaviour of $F$ near the origin. We denote by $z=(u, v)$ an arbitrary vector of $\mathbb{R}^{2}$ and suppose that:

there exists $A_{0} \in \mathcal{M}_{2}(\Omega)$ such that

$$
\lim _{|z| \rightarrow 0} \frac{2 F(x, z)-\left\langle A_{0}(x) z, z\right\rangle}{|z|^{2}}=0 \quad \text { uniformly for } x \in \Omega \text {. }
$$

Concerning the behaviour of $F$ at infinity we assume the following condition.

$$
\begin{aligned}
& \text { there exists } A_{\infty} \in \mathcal{M}_{2}(\Omega) \text { such that } \\
& \lim _{|z| \rightarrow \infty} 2 F(x, z)-\left\langle A_{\infty}(x) z, z\right\rangle=\infty \quad \text { uniformly for } x \in \Omega .
\end{aligned}
$$

In our first result we consider the resonance at the $k$ th eigenvalue. We shall prove the following theorem.

Theorem 1.1. Suppose that $\nabla F(x, 0,0) \equiv 0$ and $(F),\left(F_{0}\right),\left(F_{\infty}^{+}\right)$hold. Suppose also that $\lambda_{m}\left(A_{0}\right)<1<\lambda_{m+1}\left(A_{0}\right)$ for some $m \in \mathbb{N} \cup\{0\}$ and $1=\lambda_{k}\left(A_{\infty}\right)<$ $\lambda_{k+1}\left(A_{\infty}\right)$ for some $k \neq m$. Then there exists $\varepsilon_{0}>0$ such that problem $(P)$ has two nontrivial solutions whenever

$$
\left\langle\nabla F(x, z)-\nabla F(x, \bar{z})-A_{\infty}(x)(z-\bar{z}), z-\bar{z}\right\rangle \leq \varepsilon\|z-\bar{z}\|^{2},
$$

for any $(x, z, \bar{z}) \in \Omega \times \mathbb{R}^{2} \times \mathbb{R}^{2}$ and for some $\varepsilon<\varepsilon_{0}$.

In our second result we consider resonance at the first eigenvalue. In this case we are able to consider only a local condition at infinity. Instead of the global condition $\left(F_{\infty}^{+}\right)$, we suppose that:

there exist $A_{\infty} \in \mathcal{M}_{2}(\Omega)$, an open nonempty set $\Omega_{0} \subset \Omega$ and $M \in L^{1}(\Omega)$ such that:

(i) $\lim _{|z| \rightarrow \infty} 2 F(x, z)-\left\langle A_{\infty}(x) z, z\right\rangle=-\infty$, uniformly for $x \in \Omega_{0}$;

(ii) $2 F(x, z)-\left\langle A_{\infty}(x) z, z\right\rangle \leq M(x)$, for all $(x, z) \in \Omega \times \mathbb{R}^{2}$.

In this case our result can be stated as follows. 
Theorem 1.2. Suppose that $\nabla F(x, 0,0) \equiv 0$ and $(F),\left(F_{0}\right),\left(F_{\infty}^{-}\right)$hold. Suppose also that $\lambda_{1}\left(A_{\infty}\right)=1$ and $\lambda_{m}\left(A_{0}\right)<1<\lambda_{m+1}\left(A_{0}\right)$, for some $m \in \mathbb{N}$. Then problem $(P)$ has two nontrivial solutions.

In the proofs, we apply variational methods and Morse theory. The assumption $\left(F_{\infty}^{+}\right)$has appeared in the paper of Liu [13], where the scalar autonomous problem

$$
-\Delta u=p(u), \quad u \in H_{0}^{1}(\Omega)
$$

was considered. In that paper, the technical condition (1.2) is replaced by the assumption that $p^{\prime}(s) \leq \gamma<\lambda_{k+1}$. This kind of hypothesis is related to the LyapunovSchmidt reduction method (see [2,3]). The condition (1.2) can be translated to the scalar framework as $p^{\prime}(s) \leq \lambda_{k}+\varepsilon$ for some $\varepsilon>0$ small. We need this stronger condition because the mean value technique applied in [3] does not work in higher dimensions.

Theorem 1.1 is closely related to the result of [13] but are more general in several senses: first, we consider a system of equations; second, in [13] it was assumed that $p^{\prime}(0)<\lambda_{1}$ and here the condition $\lambda_{m}\left(A_{0}\right)<1<\lambda_{m+1}\left(A_{0}\right)$ allows the derivative at the origin to belong to any consecutive eigenvalues; finally, instead of imposing restrictions on the asymptotic limits of the function $F$, we use the more general idea of analyzing the position of the number 1 in the spectrum of the associated weighted eigenvalue problem. Hence, our approach can be used to extend the result of [13] also for the scalar case.

Since condition $\left(F_{\infty}^{-}\right)$implies that the associated functional is coercive, Theorem 1.2 is related to that proved in [11, Theorem 1.4] where the scalar equation is considered with some different hypotheses. Our results also complement those of [10], where the system $(P)$ is studied under a different condition at infinity and the nonquadratic condition introduced by Costa and Magalhães [7, 8] was assumed. This last condition is related to the conditions $\left(F_{\infty}^{ \pm}\right)$used here.

The paper is organized as follows: in the forthcoming section we present the abstract framework as well as some abstract results. The last two sections are devoted to the proofs of the theorems.

\section{Preliminaries}

Let $X$ be a real Hilbert space and $I \in C^{1}(X, \mathbb{R})$. Let $z_{0} \in X$ be an isolated critical point of $I, c=I\left(z_{0}\right)$ and $j$ be a nonnegative integer. We define the $j$ th critical group of $I$ at $z_{0}$ as being

$$
C_{j}\left(I, z_{0}\right)=H_{j}\left(I^{c}, I^{c} \backslash\left\{z_{0}\right\}\right),
$$

where $I^{c}=\{u \in X: I(u) \leq c\}$ and $H_{*}(\cdot, \cdot)$ denotes the relative singular homology group with coefficients in $\mathbb{Z}$.

We say that $I$ satisfies the Palais-Smale condition ((PS) for short) if any sequence $\left(u_{n}\right) \subset X$ such that $I^{\prime}\left(u_{n}\right) \rightarrow 0$ and $\left(\left|I\left(u_{n}\right)\right|\right) \subset \mathbb{R}$ is bounded possesses a convergent 
subsequence. If $I$ has no critical values less than $\alpha \in \mathbb{R}$ and satisfies (PS), we can define the Betti numbers

$$
\beta_{j}=\operatorname{rank} H_{j}\left(X, I^{\alpha}\right) .
$$

The (PS) condition implies that the definition of Betti numbers does not depend on $\alpha$. Moreover, there is a relation between the critical groups and the Betti numbers (see [4, Theorem I.4.3]). It reads

$$
\sum_{j=0}^{\infty} M_{j}(-1)^{j}=\sum_{j=0}^{\infty} \beta_{j}(-1)^{j}
$$

where $M_{j}=\sum_{I^{\prime}(u)=0} \operatorname{rank} C_{j}(I, u)$.

Under assumption $\left(F_{\infty}^{+}\right)$, the associated functional may not satisfy the (PS) condition. Hence, we shall apply the Lyapunov-Schmidt reduction method developed in [2] (see [13, Lemma 2.3] for the proof of the third item).

THEOREM 2.1. Let $Y$ and $W$ be closed subspaces of a separable Hilbert space $X=Y \oplus W$ and $I \in C^{1}(X, \mathbb{R})$. If there exists $\beta>0$ such that, for any $y \in Y$ and $w_{1}, w_{2} \in W$, there holds

$$
\left\langle\nabla I\left(y+w_{1}\right)-\nabla I\left(y+w_{2}\right), w_{1}-w_{2}\right\rangle \geq \beta\left\|w_{1}-w_{2}\right\|^{2},
$$

then:

(i) there exists a continuous map $\psi: Y \rightarrow W$ such that

$$
I(y+\psi(y))=\min _{w \in W} I(y+w) .
$$

Moreover, $\psi(y)$ is the unique point of $W$ such that $\langle\nabla I(y+\psi(y)), w\rangle=0$ for any $w \in W$;

(ii) the functional $\varphi: Y \rightarrow \mathbb{R}$ given by

$$
\varphi(y)=I(y+\psi(y))
$$

belongs to $C^{1}(Y, \mathbb{R})$ and $y \in Y$ is a critical point of $\varphi$ if, and only if, $y+\psi(y)$ is a critical point of $I$;

(iii) if $y \in Y$ is an isolated critical point of $\varphi$ then

$$
C_{j}(\varphi, y)=C_{j}(I, y+\psi(y)), \quad j=0,1,2, \ldots
$$

2.1. The linear problem. Hereafter we write $\int_{\Omega} u$ instead of $\int_{\Omega} u(x) d x$ and denote by $X$ be the Hilbert space $H_{0}^{1}(\Omega) \times H_{0}^{1}(\Omega)$ endowed with the inner product

$$
\langle(u, v),(\phi, \psi)\rangle=\int(\nabla u \cdot \nabla \phi+\nabla v \cdot \nabla \psi), \quad \forall(u, v),(\phi, \psi) \in X,
$$


and associated norm

$$
\|z\|^{2}=\int_{\Omega}\left(|\nabla u|^{2}+|\nabla v|^{2}\right), \quad \forall z=(u, v) \in X .
$$

By the Sobolev theorem we know that, for any $2 \leq \sigma \leq 2^{*}$ fixed, the embedding $H \hookrightarrow L^{\sigma}(\Omega) \times L^{\sigma}(\Omega)$ is continuous and therefore we can find a positive constant $S_{\sigma}$ such that

$$
\int_{\Omega}|z|^{\sigma} \leq S_{\sigma}\|z\|^{\sigma}
$$

Moreover, if $\sigma<2^{*}$, the Rellich-Kondrachov theorem implies that the above embedding is compact.

We proceed now with the study of the linear problem associated to $(P)$. We refer to [10, Section 2] for more details. We know that $\lambda$ is an eigenvalue of $(L P)$ if, and only if, $T_{A}(u, v)=\lambda^{-1}(u, v)$, where $T_{A}: X \rightarrow X$ is the symmetric bounded linear operator defined by

$$
\left\langle T_{A}(u, v),(\phi, \psi)\right\rangle=\int_{\Omega}\left\langle A(x)\left(\begin{array}{l}
u \\
v
\end{array}\right),\left(\begin{array}{l}
\phi \\
\psi
\end{array}\right)\right\rangle_{\mathbb{R}^{2}} .
$$

The first eigenvalue can be characterized as

$$
\frac{1}{\lambda_{1}(A)}=\mu_{1}(A)=\sup \left\{\left\langle T_{A} z, z\right\rangle:\|z\|=1\right\} .
$$

If $\mu_{1}(A)>0$, it can be proved (see [5,6]) that the components of the associated eigenfunction $\Phi_{1}^{A}$ are nonzero and have constant sign on $\Omega$. By using induction, if we suppose that

$$
\mu_{1}(A) \geq \mu_{2}(A) \geq \cdots \geq \mu_{k-1}(A) \geq \mu_{k}(A)>0
$$

are the $k$ first eigenvalues of $T_{A}$ with associated eigenfunctions $\left\{\Phi_{i}^{A}\right\}_{i=1}^{k}$, we can define

$$
\frac{1}{\lambda_{k+1}(A)}=\mu_{k+1}(A)=\sup \left\{\left\langle T_{A} z, z\right\rangle:\|z\|=1, z \in\left(\operatorname{span}\left\{\Phi_{1}^{A}, \ldots, \Phi_{k}^{A}\right\}\right)^{\perp}\right\} .
$$

If $\mu_{k+1}(A)>0$, then it is an eigenvalue of $T_{A}$ with associated eigenfunction $\Phi_{k+1}^{A}$ (see [9]). Moreover, if we set $Y_{k}=\operatorname{span}\left\{\Phi_{1}^{A}, \ldots, \Phi_{k}^{A}\right\}$, we have that $X=Y_{k} \oplus W_{k}$, with $W_{k}=Y_{k}^{\perp}$, and the following variational inequalities hold

$$
\|y\|^{2} \leq \lambda_{k}(A) \int_{\Omega}\langle A(x) y, y\rangle, \quad \forall y \in Y_{k},
$$

and

$$
\|w\|^{2} \geq \lambda_{k+1}(A) \int\langle A(x) w, w\rangle, \quad \forall w \in W_{k} .
$$




\section{Proof of Theorem 1.1}

In this section we prove Theorem 1.1. First of all we notice that, in view of $\left(F_{1}\right)$, the weak solutions of the problem $(P)$ are precisely the critical points of the $C^{2}$-functional $I: X \rightarrow \mathbb{R}$ given by

$$
I(z)=\frac{1}{2} \int\left(|\nabla u|^{2}+|\nabla v|^{2}\right)-\int F(x, z) .
$$

Let $A_{\infty}$ be given by $\left(F_{\infty}^{+}\right)$and assume that $\lambda_{k}\left(A_{\infty}\right)=1$. For any $1 \leq j \leq k$, let $\Phi_{j}^{A_{\infty}}$ be the normalized eigenfunction associated to the $j$ th positive eigenvalue $\lambda_{j}\left(A_{\infty}\right)$, as explained in the Section 2. If we define

$$
Y=\operatorname{span}\left\{\Phi_{1}^{A_{\infty}}, \ldots, \Phi_{k}^{A_{\infty}}\right\} \text { and } W=Y^{\perp},
$$

we have that $X=Y \oplus W$.

LEMMA 3.1. If (1.2) is verified for $\varepsilon>0$ small, then there exists $\beta>0$ such that, for any $y \in Y$ and $w_{1}, w_{2} \in W$ we have that

$$
\left\langle\nabla I\left(y+w_{1}\right)-\nabla I\left(y+w_{2}\right),\left(w_{1}-w_{2}\right)\right\rangle \geq \beta\left\|w_{1}-w_{2}\right\|^{2} .
$$

Proof. Let $J\left(v, w_{1}, w_{2}\right)$ the left-hand side of (3.1). By using (2.5), (1.2) and (2.2) we get

$$
\begin{aligned}
J\left(y, w_{1}, w_{2}\right)= & \left\|w_{1}-w_{2}\right\|^{2} \pm \int\left\langle A_{\infty}(x)\left(w_{1}-w_{2}\right), w_{1}-w_{2}\right\rangle_{\mathbb{R}^{2}} \\
& -\int\left\langle\nabla F\left(x, y+w_{1}\right)-\nabla F\left(x, y+w_{2}\right), w_{1}-w_{2}\right\rangle_{\mathbb{R}^{2}} \\
\geq & \left(1-\frac{1}{\lambda_{k+1}\left(A_{\infty}\right)}-\varepsilon S_{2}\right)\left\|w_{1}-w_{2}\right\|^{2}=\beta\left\|w_{1}-w_{2}\right\|^{2}
\end{aligned}
$$

Since $\lambda_{k+1}\left(A_{\infty}\right)>1$, it suffices to take $\varepsilon>0$ small in such way that $\beta$ is positive.

In view of the above lemma, we can use Theorem 2.1 to obtain a continuous map $\psi: Y \rightarrow W$ and a $C^{1}$-functional $\varphi: Y \rightarrow \mathbb{R}$ given by

$$
\varphi(y)=I(y+\psi(y))=\min _{w \in W} I(y+w) .
$$

Moreover, $y \in Y$ is a critical point of $\varphi$ if and only if $y+\psi(y)$ is a critical point of $I$.

Lemma 3.2. Suppose $\left(F_{\infty}\right)$ holds and $\lambda_{k}\left(A_{\infty}\right)=1$ for some $k \in \mathbb{N}$. Then the functional $\varphi$ is anti-coercive.

PROOF. Since $\lambda_{k}\left(A_{\infty}\right)=1$, we can use (3.2) and (2.4) to get, for any $y \in Y$,

$$
\begin{aligned}
2 \varphi(y) & \leq 2 I(y)=\int\left(|\nabla y|^{2}-\left\langle A_{\infty}(x) y, y\right\rangle\right)+\int\left(\left\langle A_{\infty}(x) y, y\right\rangle-2 F(x, y)\right) \\
& \leq \int\left(\left\langle A_{\infty}(x) y, y\right\rangle-2 F(x, y)\right) .
\end{aligned}
$$


Suppose that $\left(y_{n}\right) \subset Y$ is such that $\left\|y_{n}\right\| \rightarrow \infty$. Since $Y$ is finite dimensional we can set $\tilde{y}_{n}=y_{n} /\left\|y_{n}\right\|$ and suppose that $\tilde{y}_{n} \rightarrow \tilde{y} \neq 0$ strongly in $Y$. Hence, the set

$$
\widetilde{\Omega}=\{x \in \Omega:|y(x)| \neq 0\}
$$

has positive measure and $\left|y_{n}(x)\right| \rightarrow \infty$ for a.e. $x \in \widetilde{\Omega}$. The above expression, Fatou's lemma and $\left(F_{\infty}^{+}\right)$imply that

$$
2 \varphi\left(y_{n}\right) \leq C|\Omega \backslash \widetilde{\Omega}|+\int_{\widetilde{\Omega}}\left(\left\langle A_{\infty}(x) y_{n}, y_{n}\right\rangle-2 F\left(x, y_{n}\right)\right) \rightarrow-\infty \quad \text { as } n \rightarrow \infty,
$$

where $|\Omega \backslash \widetilde{\Omega}|$ denotes the Lebesgue measure of $\Omega \backslash \widetilde{\Omega}$. The lemma is proved.

We can now follow the argument of [13] to prove our first multiplicity result.

Proof of THEOREM 1.1. We first note that, since $\varphi$ is anti-coercive in the finite dimensional space $Y$, it possesses a global maximum point $y_{\max }$ such that

$$
C_{j}\left(\varphi, y_{\max }\right)= \begin{cases}\mathbb{Z} & \text { if } j=k, \\ 0 & \text { otherwise }\end{cases}
$$

Moreover, arguing as in [12, Lemma 2.1], we can prove that the Betti numbers are given by

$$
\beta_{j}= \begin{cases}1 & \text { if } j=k \\ 0 & \text { otherwise. }\end{cases}
$$

Since $\nabla I(0)=0$, it follows from Theorem 2.1(i) that $\psi(0)=0$. It follows from items (ii) and (iii) Theorem 2.1 that the origin is a critical point of $\varphi$ and $C_{*}(\varphi, 0)=$ $C_{*}(I, 0)$. In order to compute this last critical group, we observe that we can use the regularity of $F$ and some calculations to prove that the matrix $A_{0}$ given by the condition $\left(F_{0}\right)$ is precisely the second derivative $D^{2} F(x, 0)$. Thus, the condition $\lambda_{m}\left(A_{0}\right)<1<\lambda_{m+1}\left(A_{0}\right)$ implies that 0 is a nondegenerate critical point of $I$ with Morse index equals to $m$. It follows from [4, Theorem 4.1 of $\mathrm{Ch}$. 1] that

$$
C_{j}(\varphi, 0)=C_{j}(I, 0)= \begin{cases}\mathbb{Z} & \text { if } j=m \\ 0 & \text { otherwise }\end{cases}
$$

We also note that, since $m \neq k$, we have that $y_{\max } \neq 0$.

We can now argue indirectly. If 0 and $y_{\max }$ are the only critical points of $\varphi$, the expression (2.1) and the above equalities imply that

$$
(-1)^{m}+(-1)^{k}=(-1)^{k}
$$

which does not make sense. Hence, $\varphi$ has a third critical point $y_{0}$. It follows from Theorem 2.1(ii) that $y_{\max }+\psi\left(y_{\max }\right)$ and $y_{0}+\psi\left(y_{0}\right)$ are two nontrivial solutions of $(P)$. The theorem is proved. 


\section{Proof of Theorem 1.2}

In this section we prove Theorem 1.2. As we shall see, we do not need to use the reduction method in this case because, in this setting, the original functional $I$ is coercive.

Lemma 4.1. Suppose $\left(F_{\infty}^{-}\right)$hold and $\lambda_{1}\left(A_{\infty}\right)=1$. Then the functional I is coercive.

Proof. Since $\lambda_{1}\left(A_{\infty}\right)=1$, we can use (2.5) to get, for any $z \in X$,

$$
\begin{aligned}
2 I(z) & =\int\left(|\nabla z|^{2}-\left\langle A_{\infty}(x) z, z\right\rangle\right)+\int\left(\left\langle A_{\infty}(x) z, z\right\rangle-2 F(x, z)\right) \\
& \geq \int\left(\left\langle A_{\infty}(x) z, z\right\rangle-2 F(x, z)\right) .
\end{aligned}
$$

Suppose, by contradiction, that there is $\left(z_{n}\right) \subset X$ such that $\left\|z_{n}\right\| \rightarrow \infty$ and

$$
I\left(z_{n}\right) \leq C,
$$

for some $C>0$. By taking $\widetilde{z}_{n}=z_{n} /\left\|z_{n}\right\|$, we may suppose that $\tilde{z}_{n} \rightarrow \widetilde{z}$ weakly in $X$, $\tilde{z}_{n} \rightarrow \widetilde{z}$ strongly in $L^{2}(\Omega) \times L^{2}(\Omega)$ and $\widetilde{z}_{n}(x) \rightarrow \widetilde{z}(x)$ for a.e. $x \in \Omega$.

Claim. $\tilde{z}$ is an eigenfunction of the problem $(L P)$ associated to $\lambda_{1}\left(A_{\infty}\right)$.

Assuming the claim we can proceed as follows. Since $\tilde{z} \neq 0$, the same unique continuation argument employed in [14, Appendix] show that, for any open set $\widetilde{\Omega} \subset \Omega$, we have that $\widetilde{z}(x) \neq 0$ in $\widetilde{\Omega}$. Hence, taking $\Omega_{0}$ given by $\left(F_{\infty}^{+}\right)$, we concluded that $\left|z_{n}(x)\right| \rightarrow \infty$ for a.e. $x \in \Omega_{0}$. It follows from (4.1), Fatou's lemma and $\left(F_{\infty}^{-}\right)$that

$$
\begin{aligned}
2 I\left(v_{n}\right) \geq- & \int_{\Omega \backslash \Omega_{0}} M(x) d x+\int_{\Omega_{0}}\left(\left\langle A_{\infty}(x) z_{n}, z_{n}\right\rangle\right. \\
& \left.-2 F\left(x, z_{n}\right)\right) d x \rightarrow+\infty \quad \text { as } n \rightarrow \infty,
\end{aligned}
$$

which contradicts (4.2). Thus, $I$ is coercive.

In order to prove the claim we first note that

$$
\limsup _{n \rightarrow \infty} \frac{1}{\left\|z_{n}\right\|^{2}} \int_{\Omega}\left(\left\langle A_{\infty}(x) z_{n}, z_{n}\right\rangle-2 F\left(x, z_{n}\right)\right) \geq 0 .
$$

On the other hand,

$$
\int\left\langle A_{\infty}(x) \tilde{z}_{n}, \tilde{z}_{n}\right\rangle=1-\frac{2}{\left\|z_{n}\right\|^{2}} I\left(z_{n}\right)+\frac{1}{\left\|z_{n}\right\|^{2}} \int\left(\left\langle A_{\infty}(x) z_{n}, z_{n}\right\rangle-2 F\left(x, z_{n}\right)\right) .
$$

Taking the limit and using (4.2) we get

$$
\int\left\langle A_{\infty}(x) \widetilde{z}, \widetilde{z}\right\rangle \geq 1
$$


Since $\widetilde{z}$ is the weak limit of $\widetilde{z}_{n}$ we have that $\|\widetilde{z}\| \leq 1$. Hence, it follows from (2.5) that

$$
1 \geq\|\widetilde{z}\|^{2} \geq \int\left\langle A_{\infty}(x) \tilde{z}, \tilde{z}\right\rangle \geq 1 .
$$

This and (2.3) prove the claim.

We are now ready to prove our last multiplicity result.

PROOF OF THEOREM 1.2. As in the proof of Theorem 1.1 the critical groups of $I$ at the origin are

$$
C_{j}(I, 0)= \begin{cases}\mathbb{Z} & \text { if } j=m, \\ 0 & \text { otherwise. }\end{cases}
$$

Since $I$ is coercive, it satisfies the (PS) condition. So we can minimize $I$ and we obtain a global minimum point $z_{\min }$ satisfying

$$
C_{j}\left(I, z_{\min }\right)= \begin{cases}\mathbb{Z} & \text { if } j=0, \\ 0 & \text { otherwise. }\end{cases}
$$

In particular, recalling that $m \neq 0$, we conclude that $z_{\min } \neq 0$. Moreover, for $\alpha>0$ sufficiently large we have $I^{-\alpha}=\varnothing$. So, $H_{*}\left(X, I^{-\alpha}\right)=H_{*}(X)$ and therefore the Betti numbers are

$$
\beta_{j}= \begin{cases}1 & \text { if } j=0, \\ 0 & \text { otherwise. }\end{cases}
$$

If 0 and $z_{\min }$ are the only critical points of $I$, we can use the expression (2.1) and the above equalities to get

$$
(-1)^{m}+(-1)^{0}=(-1)^{0},
$$

which does not make sense. Hence, $I$ has at least three critical points and the theorem is proved.

\section{References}

[1] T. Bartsch and S. J. Li, 'Critical point theory for asymptotically quadratic functionals and applications to problems with resonance', Nonlinear Anal. 28 (1997), 419-441.

[2] A. Castro, 'Reduction methods via minimax', in: Differential Equations (São Paulo, 1981), Lecture Notes in Mathematics, 957 (Springer, Berlin, 1982), pp. 1-20.

[3] A. Castro and J. Cossio, 'Multiple solutions for a nonlinear Dirichlet problems', SIAM J. Math. Anal. 25 (1994), 1554-1561.

[4] K. C. Chang, Infinite Dimensional Morse Theory and Multiple Solution Problems (Birkhäuser, Boston, MA, 1993).

[5] K. C. Chang, 'An extension of the Hess-Kato theorem to elliptic systems and its applications to multiple solutions problems', Acta Math. Sin. 15 (1999), 439-454.

[6] K. C. Chang, 'Principal eigenvalue for weigth in elliptic systems', Nonlinear Anal. 46 (2001), 419-433.

[7] D. G. Costa and C. A. Magalhães, 'Variational elliptic problems which are nonquadratic at infinity', Nonlinear Anal. 23 (1994), 1401-1412. 
[8] D. G. Costa and C. A. Magalhães, 'A unified approach to a class of strongly indefinite functionals', J. Differential Equations 125 (1996), 521-547.

[9] D. G. deFigueiredo, 'Positive solutions of semilinear elliptic problems', in: Differential Equations (São Paulo, 1981), Lecture Notes in Mathematics, 957 (Springer, Berlin, 1982), pp. 34-87.

[10] M. F. Furtado and F. O. V. de Paiva, 'Multiplicity of solutions for resonant elliptic systems', J. Math. Anal. Appl. 319 (2006), 435-449.

[11] M. F. Furtado and E. A. B. Silva, 'Double resonant problems which are locally nonquadratic at infinity', Electron. J. Differ. Equ., Conf. 6 (2001), 155-171.

[12] S. J. Li and S. Liu, 'Critical groups at infinity, saddle point reduction and elliptic resonant problems', Commun. Comtem. Math. 5 (2003), 761-773.

[13] S. Liu, 'Remarks on multiple solutions for elliptic resonant problems', J. Math. Anal. Appl. 336 (2007), 498-505.

[14] O. Lopes, 'Radial symmetry of minimizers for some translation and rotation invariant functionals', J. Differential Equations 124 (1996), 378-388.

MARCELO F. FURTADO, Departamento de Matemática, Universidade de Brasília, 70910-900, Brasília - DF, Brazil

e-mail: mfurtado@unb.br

FRANCISCO O. V. DE PAIVA, IMECC-UNICAMP, Caixa Postal 6065, 13081-970, Campinas-SP, Brazil

e-mail: odair@ime.unicamp.br 